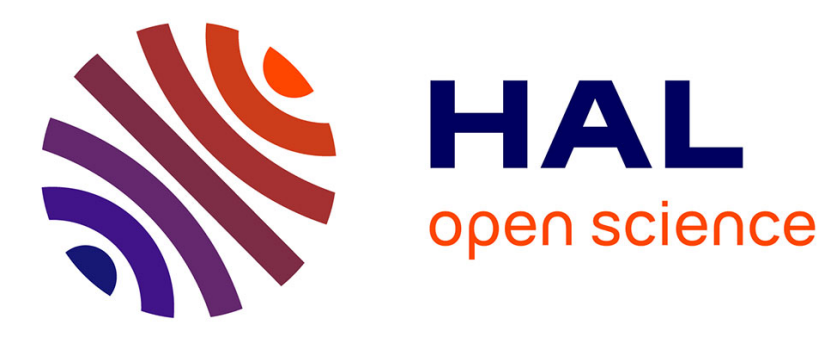

\title{
Conditions of microvessel occlusion for blood coagulation in flow
}

Anass Bouchnita, T Galochkina, P Kurbatova, Patrice Nony, Vitaly Volpert

\section{To cite this version:}

Anass Bouchnita, T Galochkina, P Kurbatova, Patrice Nony, Vitaly Volpert. Conditions of microvessel occlusion for blood coagulation in flow. International Journal for Numerical Methods in Biomedical Engineering, 2017, CMBE15: SELECTED PAPERS FROM THE 4TH INTERNATIONAL CONFERENCE ON COMPUTATIONAL \& MATHEMATICAL BIOMEDICAL ENGINEERING 2015, 10.1002/cnm.2850 . hal-01573775

\section{HAL Id: hal-01573775 \\ https://hal.science/hal-01573775}

Submitted on 10 Aug 2017

HAL is a multi-disciplinary open access archive for the deposit and dissemination of scientific research documents, whether they are published or not. The documents may come from teaching and research institutions in France or abroad, or from public or private research centers.
L'archive ouverte pluridisciplinaire HAL, est destinée au dépôt et à la diffusion de documents scientifiques de niveau recherche, publiés ou non, émanant des établissements d'enseignement et de recherche français ou étrangers, des laboratoires publics ou privés. 


\title{
Conditions of vessel occlusion for blood coagulation in flow
}

\author{
A. Bouchnita ${ }^{1,2,3, *}$ T. Galochkina ${ }^{1,4,5}$, P. Kurbatova $^{1,2}$, P. Nony $^{2,6}$ and V. Volpert ${ }^{1}$ \\ ${ }^{1}$ Institut Camille Jordan, UMR 5208 CNRS, University Lyon 1, 69622 Villeurbanne, France \\ ${ }^{2}$ Laboratoire de Biométrie et Biologie Evolutive, UMR 5558 CNRS, University Lyon 1, 69376 Lyon, France \\ ${ }^{3}$ Laboratory of Study and Research in Applied Mathematics, Mohammadia School of Engineers, Mohamed V \\ university, Rabat, Morocco \\ ${ }^{4}$ Department of Biophysics, Faculty of Biology, M.V. Lomonosov Moscow State University, Leninskie gory 1, \\ Moscow, Russia \\ ${ }^{5}$ Federal Research Clinical Center of Federal Medical \& Biological Agency of Russia, Orekhovy boulevard 28, \\ Moscow, Russia \\ ${ }^{6}$ Hospices Civils de Lyon, Service de Pharmacologie Clinique, Lyon, France
}

\section{SUMMARY}

Vessel occlusion is a perturbation of blood flow inside a blood vessel because of the fibrin clot formation. As a result, blood circulation in the vessel can be slowed down or even stopped provoking the risk of cardiovascular events. In order to explore this phenomenon, we suggest a mathematical model of blood clotting. We describe the concentrations of blood factors with reaction-diffusion system of equations, blood flow is modelled with the Navier-Stokes equations with clot considered as a porous medium. We identify the conditions of partial or complete occlusion in a small vessel depending on various physical and physiological parameters. The obtained results are then compared with the analytical conditions on the arterial occlusion for the simplified mathematical model. We observe different regimes of vessel occlusion depending on the model parameters both for numerical simulations and in theoretical study. Copyright (c) 2015 John Wiley \& Sons, Ltd.

Received ..

KEY WORDS: blood coagulation; vascular occlusion; Navier-Stokes equations; reaction-diffusion system 


\section{INTRODUCTION}

\subsection{Blood coagulation and vascular occlusion}

Clot formation is initiated by the damage of the endothelial tissue at the internal surface of blood vessel walls. As the result, tissue factor (TF) that is normally isolated from blood plasma, is bared and forms a complex with factor VII. This complex activates factors IX and X that initiate the coagulation cascade. The key stage of this process is formation of thrombin that accelerates the reaction of conversion of fibrinogen into fibrin. The latter forms fibrin polymer which together with platelets constitutes the clot. Once initiated by activated factors IX and X, thrombin production is self-sustained through the positive feedback loops of the coagulation cascade.

Two main physiological mechanisms control the thrombin production preventing spontaneous clotting: thrombin plasma inhibitors and activated protein $\mathrm{C}$ pathway. One of the most important thrombin inhibitors is antithrombin that binds thrombin molecules. Activation of protein $\mathrm{C}$ takes place due to its reaction with thrombin-thrombomodulin complex that is formed at the surface of the endothelial tissue. The active form of protein C (APC) then inhibits the amplification phase of thrombin generation [1]. One more significant factor determining the dynamics of clot formation is the role of blood flow. From one side the flow removes the substances participating in thrombin production from the clot. From the other side, it also transports the activated protein $\mathrm{C}$ generated in proximity of intact tissues to the region of the wound. The balance between the physiological mechanisms of thrombin production and clot growth regulation determines different regimes of vessel occlusion under normal conditions as well as in pathological situations (thrombosis or hemophilia). In the current study we will determine the conditions of different regimes of blood coagulation and clot growth on the basis of mathematical modelling.

\footnotetext{
$\overline{{ }^{*} \text { Correspondence }}$ to: Anass Bouchnita, Institut Camille Jordan, UMR 5208 CNRS, University Lyon 1, 69622 Villeurbanne, France.

${ }^{\dagger}$ E-mail: anass.bouchnita@univ-lyon1.fr 


\subsection{Mathematical modelling of blood coagulation}

Various approaches are employed to study clot growth. Equations of chemical kinetics are used to describe reactions between the coagulation factors [2, 3], platelet activation and formation of platelets plug [4]. Reaction-diffusion equations for the concentrations of blood factors take into account their spatial distribution $[5,6]$. These distributions are influenced by hemodynamics. Blood flow can be modelled with partial differential equations or with particle methods. Navier-Stokes equations are used if blood is considered as a Newtonian fluid [7, 8], and various viscoelastic models if non-Newtonian properties of blood are taken into account $[9,7]$.

Clot growth can be modelled as a free boundary problem where the interface separates blood flow from the clot region [10]. Another approach considers blood flow and clot as a continuous medium where clot is described as a high viscosity or a non-Newtonian fluid [11, 7]. Clot can also be described as a porous medium with an additional term in the Navier-Stokes equations [12] describing fluid velocity deceleration inside the clot.

Most of these works consider only the case of prescribed flow velocity at the inlet of the vessel and do not consider the case of pressure driven flow. Below we demonstrate that these two cases are essentially different because complete vessel occlusion can occur only in case of pressure driven blood flow model.

In order to model platelets, both particle and continuous methods were used. Among the particle methods, the immersed boundary method was used in [13], cellular Potts model [14], dissipative particle dynamics in $[9,15,16,17]$. Advection-diffusion-reaction were also used to model platelet concentration (in their active and non-active forms) [4]. In hybrid models, particle methods are used to describe blood flow and blood cells and PDEs to describe concentrations of blood factors [16, 18]. It is also possible to model blood flow with the Navier-Stokes equations and the coagulation process with cellular automata [14]. 


\subsection{Objectives of the paper}

In the current work we study the conditions of complete or partial occlusion in small vessels (arterial or venous thrombosis). We develop a computational model describing blood coagulation and clot growth (Section 2). The model consists of the reaction-diffusion system of equations for blood factor concentrations coupled with the Navier-Stokes equations modelling blood flow. Platelets are not included in the model and will be studied in a future work. We also consider a simplified model that admits an analytical investigation (Section 4). This model is a simplified approximation of the first one. It consists of a scalar 1D reaction- diffusion equation.

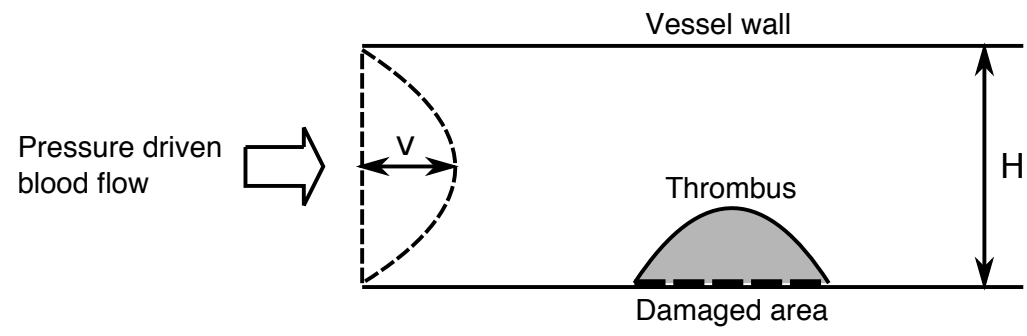

Figure 1. Schematic representation of the computational domain. It corresponds to a section of a blood vessel. A part of the lower boundary (damaged wall) initiates clot growth.

We use both complete and simplified model to show the existence of different conditions of vessel occlusion. Furthermore, we explain the underlying mechanisms related to the initiation, amplification, propagation and inhibition of blood clotting in quiescent plasma and in blood flow. We pay particular attention to the role of blood flow in the dynamics of clot formation. We analyze the evolution of the clot growth under the flow conditions and the influence of the clot on the blood flow in the numerical simulations (Section 3). We also investigate the influence of the different parameters such as sheer rate, lumen diameter and wound area length on the blood flow velocity and clot growth. The possible explanations of the observed dependencies are proposed on the basis of the simplified model. 


\section{MATHEMATICAL MODELLING OF CLOT DEVELOPMENT IN BLOOD FLOW}

In this section we formulate the model of blood coagulation and clot growth in a 2D rectangular domain. We use reaction-diffusion equations with convection terms to describe blood factor concentration dynamics in plasma. Navier-Stokes equations are used to simulate blood flow. Clot is considered as a porous medium where flow velocity is described with the Navier-Stokes equations with additional terms depending on the clot porosity. This approach allows us to describe blood flow and clot growth by the same model without tracking the clot geometry.

\subsection{Blood factors concentrations}

We consider a two-dimensional rectangular domain that corresponds to a section of blood vessel (Figure 1). Blood flow crosses it along the axial direction. A part of the vessel boundary exposes tissue factor (damaged wall) and initiates blood coagulation. We consider a reaction-diffusion system of equations [19] for the concentrations of prothrombin $P$, thrombin $T$, antithrombin $A$, factors IX and $\mathrm{X}$ with their total concentration denoted by $B$ and their active form $B_{a}$, protein $\mathrm{C}$ denoted by $C$ and its active form $C_{a}$, fibrinogen $F_{g}$, fibrin $F$ and fibrin polymer $F_{p}$ :

$$
\begin{gathered}
\frac{\partial P}{\partial t}+\nabla \cdot(v P)=D \Delta P-F\left(T, B_{a}, C_{a}\right) P, \\
\frac{\partial T}{\partial t}+\nabla \cdot(v T)=D \Delta T+F\left(T, B_{a}, C_{a}\right) P-k_{a} A T, \\
\frac{\partial A}{\partial t}+\nabla \cdot(v T)=D \Delta A-k_{a} A T, \\
\frac{\partial B}{\partial t}+\nabla \cdot(v B)=D \Delta B-k_{b} B, \quad \frac{\partial B_{a}}{\partial t}+\nabla \cdot\left(v B_{a}\right)=D \Delta B_{a}-k_{b} B_{a}, \\
\frac{\partial C}{\partial t}+\nabla \cdot(v C)=D \Delta C-k_{c} C, \quad \frac{\partial C_{a}}{\partial t}+\nabla \cdot\left(v C_{a}\right)=D \Delta C_{a}-k_{c} C_{a},
\end{gathered}
$$




$$
\begin{gathered}
\frac{\partial F_{g}}{\partial t}+\nabla \cdot\left(v F_{g}\right)=D \Delta F_{g}-k_{1} T F_{g}, \\
\frac{\partial F}{\partial t}+\nabla \cdot(v F)=D \Delta F+k_{1} T F_{g}-k_{2} F, \\
\frac{\partial F_{p}}{\partial t}=k_{2} F .
\end{gathered}
$$

Here $v$ is the flow velocity, $D$ is the diffusion coefficient taken the same for all concentrations except for fibrin polymer. Fibrin polymer forms a solid clot, and thus does not diffuse and is not transported by the flow. The rate of thrombin production in equations (1), (2) is given by the following function (see [19], supplementary materials):

$$
F\left(T, B_{a}, C_{a}\right)=k_{3} B_{a}+\frac{k_{4} T^{3}}{1+k_{5} C_{a}}
$$

In this expression, the first term in the right-hand side corresponds to the initiation phase. The second term represents the propagation phase where thrombin generation depends on its own concentration. Activated protein $\mathrm{C}$ decreases the reaction rate. More complete kinetics of thrombin production includes other reactions and blood factors. It can be reduced to a one-step reaction under the approximation of detailed equilibrium (fast reactions).

\subsection{Boundary conditions}

We describe the initiation of coagulation cascade as the reaction of activation of factors IX and X $(B)$ by the complex of TF and factor VII formed on the damaged surface $\left(T_{F}^{*}\right)$. The inactive forms of factors IX and $\mathrm{X}$ are present in the bulk solution, their activation takes place through the formation of complex with $\mathrm{TF}^{*}\left(\left[T_{F}^{*} B\right]\right)$. The concentration of this surface complex satisfies the following equation:

$$
\frac{\partial\left[T_{F}^{*} B\right]}{\partial n}=k_{7}^{+} B\left(T_{F}^{*}-\left[T_{F}^{*} B\right]\right)-k_{7}^{-}\left[T_{F}^{*} B\right],
$$


where the first term in the right-hand side of this equation describes the flux of $B$ to the surface, the second term describes the flux from the surface. Assuming that this reaction is fast, we can use the detailed equilibrium

$$
k_{7}^{+} B\left(T_{F}^{*}-\left[T_{F}^{*} B\right]\right)=k_{7}^{-}\left[T_{F}^{*} B\right] .
$$

Then

$$
\left[T_{F}^{*} B\right]=\frac{k_{7} B T_{F}^{*}}{1+k_{7} B}
$$

where $k_{7}=k_{7}^{+} / k_{7}^{-}$.

The boundary conditions for the variables $B$ and $B_{a}$ at the damaged surface are:

$$
\frac{\partial B}{\partial n}=-k_{7}^{+} B\left(T_{F}^{*}-\left[T_{F}^{*} B\right]\right), \quad \frac{\partial B_{a}}{\partial n}=k_{7}^{-}\left[T_{F}^{*} B\right],
$$

We prescribe the zero flux condition at the intact surface.

In order to simplify the boundary conditions, let us introduce the variable $z=B+B_{a}$. From equations (10) and the boundary conditions for $B$ and $B_{a}$ we get:

$$
\frac{\partial z}{\partial t}+\nabla \cdot(v z)=D \Delta z-k_{b} z, \quad \frac{\partial z}{\partial n}=0 .
$$

Assuming that initially $B+B_{a}=B^{0}$, where $B^{0}$ is the initial concentration of factors IX and X, we conclude that the solution of problem (10) is identically constant, $B+B_{a}=B^{0}$. Hence we can exclude the variable $B$ from the equations and from the boundary condition:

$$
\left.\frac{\partial B_{a}}{\partial n}\right|_{\Gamma_{d}}=\frac{k_{6}\left(B^{0}-B_{a}\right)}{1+k_{7}\left(B^{0}-B_{a}\right)}
$$

where $k_{6}=k_{7}^{-} k_{7} T_{F}^{*}$.

Protein $\mathrm{C}$ is activated by the complex of thrombin-thrombomodulin. The fluxes of protein $\mathrm{C}$ at the surface in its active and inactive forms are given by the following boundary conditions:

$$
\frac{\partial C}{\partial n}=-k_{8}^{+} C\left(\left(T T_{m}\right)-\left(C T T_{m}\right)\right), \quad \frac{\partial C_{a}}{\partial n}=k_{8}^{-}\left(C T T_{m}\right) .
$$


Here $T_{m}$ is the concentration of thrombomodulin present at the undamaged surface. Protein $\mathrm{C}$ arrives next to thrombin and thrombomodulin and forms the complex $\left(C T T_{m}\right)$. Next, $k_{8}^{+}$is the reaction rate constant of protein $\mathrm{C}$ activation by the complex of thrombin thrombomodulin, $k_{8}^{-}$is the rate of the inverse reaction. Again using the detailed equilibrium, we obtain the condition of APC production on undamaged walls:

$$
\begin{gathered}
\left.\frac{\partial C_{a}}{\partial n}\right|_{\Gamma_{u}}=\frac{k_{8}\left(C^{0}-C\right)\left[T T_{m}\right]}{1+k_{9}\left(C^{0}-C_{a}\right)}, \\
{\left[T T_{m}\right]=\frac{k_{T} T T_{m}}{1+k_{T} T T_{m}},}
\end{gathered}
$$

where $\left[T T_{m}\right]$ is the complex thrombin-thrombomodulin that activates protein $\mathrm{C}$ present in blood plasma. We consider the no-flux boundary conditions for these concentrations at the other parts of the boundary and also for other concentrations. In the case of nonzero flow velocity, the concentrations of prothrombin, fibrinogen and antithrombin at the entrance of the domain $(x=0)$ are kept constant.

\subsection{Blood flow model}

We use the Navier-Stokes equations to describe the blood flow. Non-Newtonian properties of blood are not taken into account. We model clot as a porous medium with the porosity depending on the concentration of fibrin polymer [20]. The flow through the clot is modelled by the Navier-Stokes equations with additional terms which describe the fluid deceleration by the porous medium. The advantage of this approach is that we use the same model in the whole computational domain. We do not need to determine the clot geometry and to solve Navier-Stokes equations in the outer (with respect to the clot) domain. We have the following equations for the incompressible fluid flow:

$$
\begin{gathered}
\frac{\partial \vec{u}}{\partial t}+\vec{u} \nabla \vec{u}=-\nabla p+\frac{1}{R e} \Delta \vec{u}-\frac{1}{K_{f}(\vec{x})} \vec{u}, \\
\nabla \vec{u}=0,
\end{gathered}
$$

where $\vec{u}=\left(u_{x}, u_{y}\right)$ is the velocity vector, $p$ is the pressure, $R e=\frac{\|u\| H}{\nu}$ is the Reynolds number determined by the velocity norm $\|u\|=\sqrt{u_{x}^{2}+u_{y}^{2}}$, the kinematic viscosity $\nu$ and the height of the 
vessel $H$. In this work we consider laminar flows with $R e=300 . K_{f}$ is the hydraulic permeability of fibrin polymer [20]:

$$
\frac{1}{K_{f}(\vec{x})}=\alpha^{2} 16 F_{p}(\vec{x})^{1.5}\left(1+56 F_{p}(\vec{x})^{3}\right) .
$$

Here $\alpha$ is the fiber radius.

We prescribe the no-slip boundary conditions for velocity at the upper and lower walls, and periodic boundary conditions in the axial direction. Blood flow is driven by the pressure difference. Therefore, we use periodic boundary conditions for velocity coupled with constant pressure difference condition:

$$
p_{\text {in }}=p_{\text {out }}+\Delta p
$$

where $p_{i n}$ is the pressure at the inlet, $p_{\text {out }}$ is the outlet pressure and $\Delta p$ is the pressure difference which is considered as an imposed constant. Contrary to the condition of fixed inlet velocity, this imposed pressure difference condition allows the clot to completely occlude the vessel and obstruct the flow. This condition is verified in experimental settings [21] as well as in some areas of the circulatory system such as capillary networks.

\subsection{Numerical implementation}

Reaction-diffusion system of equation is solved numerically with a finite difference method and an upwinding method in discretizing convective terms in order to avoid numerical instabilities in the convection dominated cases. A regular $2000 \times 400$ mesh is used in the numerical simulations. Accuracy of the results was controlled by decreasing the time and space steps.

Numerical implementation of the Navier-Stokes equations is carried out with the projection method [22], and Successive Over Relaxation (SOR) method is used to solve the pressure Poisson problem. We present the details of the numerical implementation in the appendix. 


\section{NUMERICAL SIMULATIONS OF VESSEL OCCLUSION}

\subsection{Blood coagulation in quiescent plasma}

We can determine three main regimes of the clot growth in quiescent plasma [19]. The first one corresponds to the initiation of coagulation cascade with rather fable stimuli leading to the production of small amount of thrombin yet not sufficient to launch the propagation phase and thus quickly suppressed by the action of plasma inhibitors. As the result, only a small amount of fibrin polymer is produced near the injury. The second regime is characterized by self-sustained production of thrombin. The propagation of thrombin concentration is arrested through the activated protein $\mathrm{C}$ pathway that prevents the unlimited clot growth. The resulting clot height reaches some limit that is less than the vessel diameter. In the third regime inhibition by APC is not sufficient to stop clot growth and in case of bounded vessel, clot fills the whole cross section and cause complete vessel occlusion. In terms of mathematical problem on the half plane, this case corresponds to the unlimited propagation of the reaction-diffusion wave.

The regime of thrombus development depends on a number of physical and physiological parameters such as the damaged area width, tissue factor and thrombomodulin concentrations as well as on the concentration of antithrombin. We used our model to analyze how the final clot height depends on the widths of the wound area in case coagulation takes place in quiescent plasma (Figure 2, a) and blood flow. In case of quiescent plasma the complete occlusion of the vessel is observed when the size of the damaged area exceeds $6 \mu m(H=50 \mu m)$. Otherwise, a smaller clot of bounded size $(H<50 \mathrm{~m})$ is formed near the vessel wall.

Thus, in our model a complete vessel occlusion by the clot occurs only if the damaged area is sufficiently wide. This result can be explained by the details of APC distribution in plasma. Protein $\mathrm{C}$ is activated at the surface outside the wounded area and then it diffuses towards the injury site, where the coagulation reactions take place and suppresses the thrombin production. If the wounded area is wide enough, then APC diffusion will not be sufficient to cover the whole region of thrombin production. 

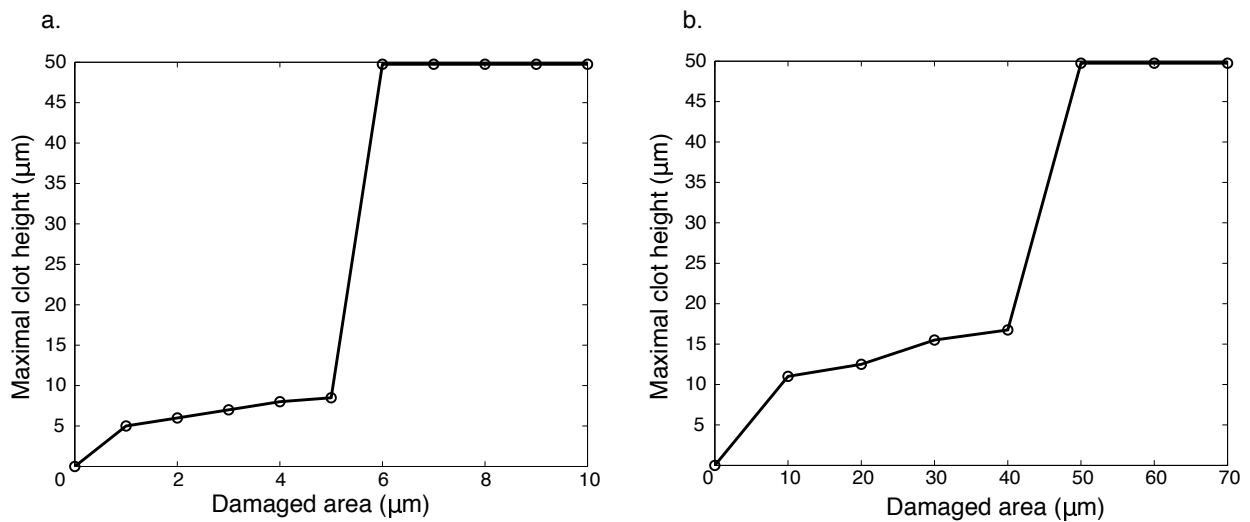

Figure 2. The final height of the clot for different damaged area widths: a) in quiescent plasma, b) in blood flow (b). In both cases there is a sharp transition from the partial occlusion regime to the complete occlusion as the clot width exceeds some critical value. This critical value is much larger for blood coagulation in flow.

\subsection{Blood coagulation in flow}

Blood flow influences clot growth in multiple ways. It brings fibrinogen and inactivated blood factors to the growing clot accelerating chemical reactions. At the same time blood flow removes thrombin and thus prevents thrombin accumulation due to the positive feedback loops of the coagulation cascade and thus slows down clot growth. Finally, blood flow transports APC generated at the intact surface to the wound region thus contributing to the downregulation of the thrombin production.

At the same time, growing clot modifies the blood flow dynamics. In our computational model this influence depends significantly on the conditions imposed on the flow velocity at the boundaries of the considered domain. Let us consider first the case where the fluid velocity at the entrance of the computational domain is time independent. Since fluid velocity decreases inside clot, then due to the mass conservation law the velocity would increase in the part of the cross section of the vessel without clot. The resulting high fluid velocity prevents clot growth removing thrombin and other blood factors participating in coagulation reactions. If we consider the limiting case where the clot has zero porosity and the fluid velocity inside the clot is zero, then complete vessel occlusion is not possible since fluid should leave the computational domain. 
The situation is different in the case of a pressure driven flow where the fluid velocity at the entrance is not fixed and the total flow rate can decrease due to the hydrodynamic resistance exerted by the clot. For a given pressure difference, the fluid velocity can decrease and the total vessel occlusion becomes possible. In this case there are two competing factors determining the blood flow dynamics. Flow velocity increases in the narrow part of the vessel, and the total flow rate decreases because of the clot resistance. It appears that the first factor is more important in the case where the width of the clot is small, while the second one becomes more important in case of large clot.
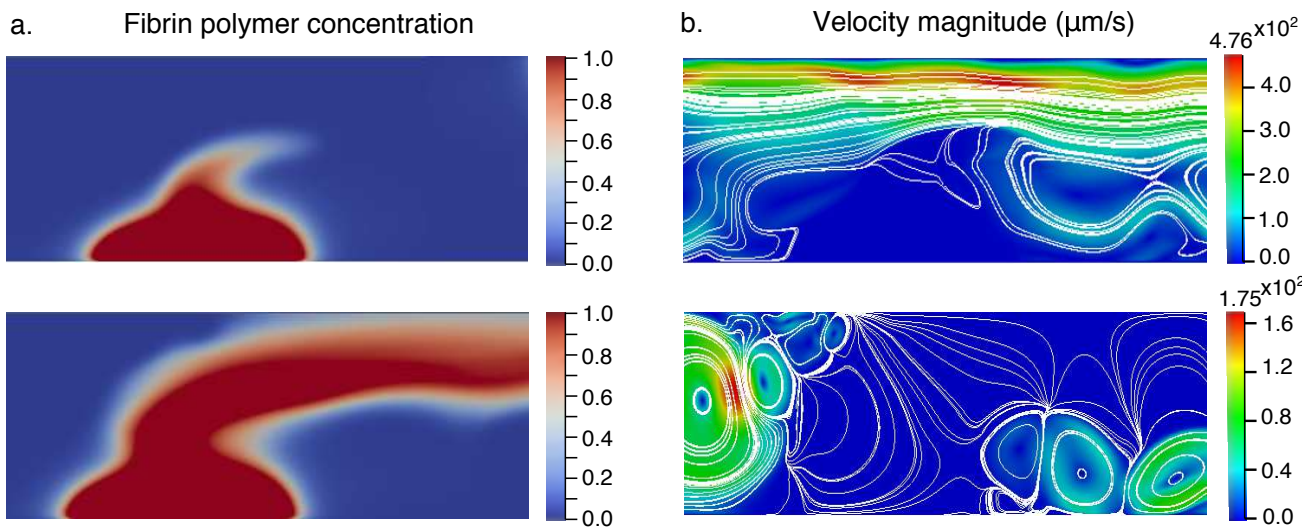

Figure 3. Snapshots of simulations with complete occlusion. Blood flow is not sufficient to stop clot growth (initial velocity is $v=200 \mu \mathrm{m} / \mathrm{s}$ ). APC does not allow clot growth width but it still grows across the flow. a) Fibrin polymer concentrations are shown at two consecutive moments of time. b) The corresponding flow velocity fields are shown with the velocity value (color scale) and streamlines (white curves).

The described interplay between the dynamics of the clot growth and the distribution of the blood flow velocity is also observed in our computational model. Here we consider the case when the thrombomodulin concentration is large enough to generate a sufficient amount of APC that suppresses clot expansion in the axial direction. If we take the blood flow velocity rather low, then the thrombin wave still propagates to the top despite the inhibition by APC. As the result, the clot occludes the vessel and completely obstructs blood flow (Figure 3, a). It is important to note that the fast development of the clot can lead to the emergence of vortices (Figure 3, b). 

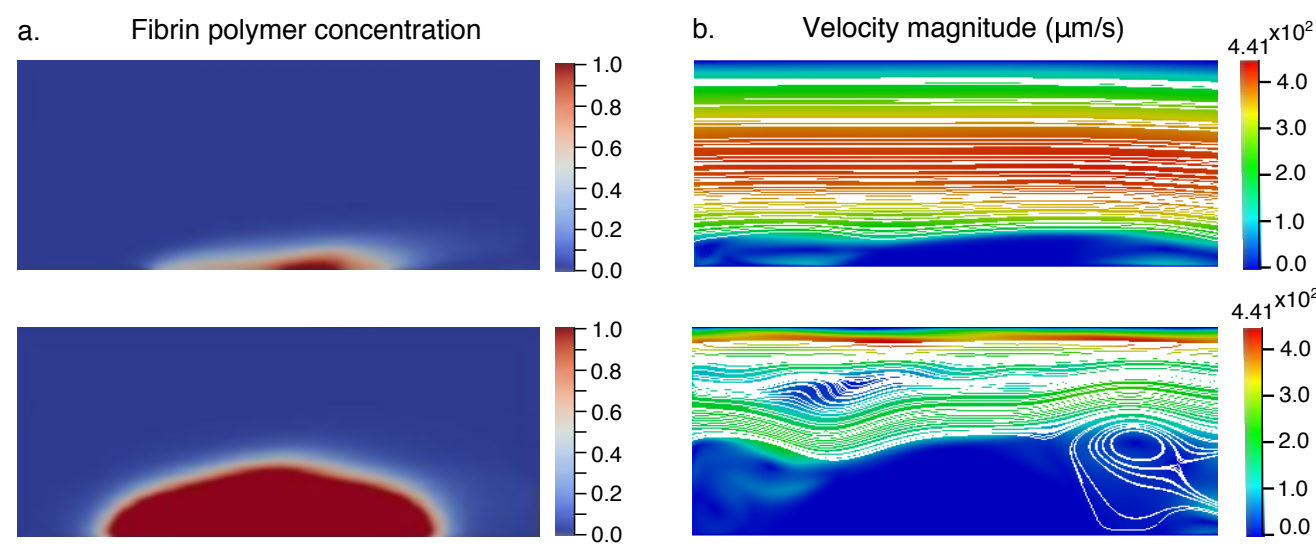

Figure 4. Two snapshots of the simulation with partial occlusion. The initial flow velocity at the entrance of the vessel is $v=400 \mu \mathrm{m} / \mathrm{s}$. a) Fibrin polymer concentrations, b) Blood flow velocity magnitude is shown with a color scale and streamlines as white curves.

If the initial blood flow velocity is sufficiently high, then it stops clot growth (Figure 4). Blood flow transports APC to the wounded area already at the very beginning of the clot formation and thus slows down thrombin production, especially at the part of the damaged area closer to the coming flow (left part of the injury in our computational domain, Figure 4, a). After some time the accumulated concentration of thrombin is sufficient to accelerate its self-sustained production and clot development. Clot growth is stopped by blood flow when clot height approaches to the half of the vessel diameter (Figure 4, b). The observed regime corresponds to the partial vessel occlusion.

The main parameters that determine the final clot size are the width of damaged area and the initial flow velocity. The dependence of the final clot size on the damaged area width for the model with the flow (Figure 2, b) is qualitatively similar to the case of quiescent plasma (Figure 2, a). If the damaged area width is large enough, then the clot continue growing till it reaches the upper vessel wall thus causing a complete vessel occlusion. If the width is sufficiently small, the final clot size remains less than the vessel diameter. These two regimes are separated with a narrow transition zone. Let us note that this transition occurs for $40-50 \mu m$ of the wound width in the case of model with the flow while for the model without flow the threshold damaged area width is only about $5-6$ 
$\mu m$. Hence we can conclude that blood flow provides an important additional (with respect to APC) mechanism that can stop clot growth.

a.

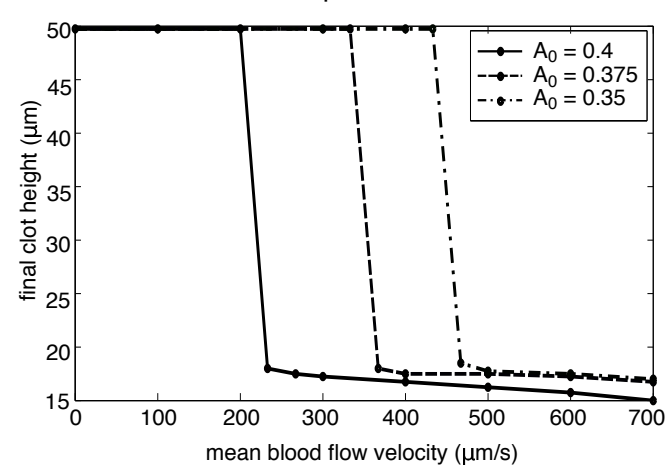

b. Simplified model

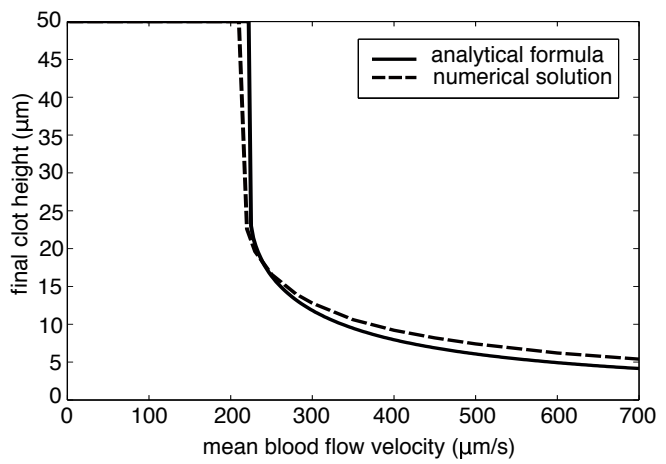

Figure 5. The final height of the clot in case of different values of the initial blood flow velocity. a): numerical simulations for three different values of antithrombin concentration in plasma $\left(A_{0}\right)$. b): numerical (solid line) and analytical (dashed line) solutions of the simplified model for $A_{0}=0.4$. For the complete and simplified models we observe complete occlusion for low flow velocities and partial occlusion for high velocities with a sharp transition between these two regimes.

The dependence of the final clot size on the initial flow velocity is shown in Figure 5, a. The complete vessel occlusion occurs only if the flow velocity is small enough. If the flow velocity is sufficiently large, then the size of the clot remains bounded (partial occlusion). We again observe a sharp transition between these two regimes for our model.

Thus, the observed three regimes of clot growth: initiation without propagation, initiation with limited propagation (partial occlusion), and initiation with unlimited propagation (complete occlusion) are qualitatively similar for the clot growth in quiescent plasma and in the blood flow. In the next section we will determine the conditions of the existence of each of these three regimes on the basis of the simplified theoretical model. 


\section{SIMPLIFIED ANALYTICAL MODEL OF VESSEL OCCLUSION}

\subsection{Thrombin distribution and initiation of clot growth}

Let us consider the 1D model describing the thrombin distribution in the vertical cross section of the 2D model:

$$
\frac{\partial T}{\partial t}=D \frac{\partial^{2} T}{\partial y^{2}}+\Phi(T, y)
$$

where

$$
\Phi(T, y)=\left(k_{1} B_{a}(y)+k_{2} T^{3}\right)\left(T^{0}-T\right)-\sigma(y) T,
$$

$D$ is the diffusion coefficient, $T^{0}$ is the prothrombin concentration initially present in blood flow, $k_{1}$ and $k_{2}$ are kinetic coefficients. The function $B_{a}(y)$ expresses the initiation of thrombin generation by factors IXa and Xa. It corresponds to a stationary solution of the equation (10), $B_{a}(y)=\lambda e^{-y / \sigma}$.

The coefficient $\sigma$ represents inhibition of thrombin production by antithrombin and blood flow. We set $\sigma=\alpha_{1} A_{0}+\alpha_{2} v(y)$, where $A$ is the antithrombin concentration supposed to be constant, and $v(y)$ is blood flow velocity in the horizontal direction. Inhibition by APC can be taken into account in the nonlinear reaction term. For simplicity, we do not consider it here.

This equation is obtained as an approximation of the model presented in Section 2 where equations (1)-(5) are replaced by the single equation for the thrombin concentration under the assumption of detailed equilibrium (fast reactions). Equations (6)-(8) are decoupled, and the corresponding concentrations do not directly influence thrombin production. The flow velocity is replaced by an approximate solution of the Navier-Stokes equations specified below.

If $k_{1}=\alpha_{2}=0$, then the function $\Phi$ does not depend on $y$, and (17) is an autonomous reactiondiffusion equation. It has a travelling wave solution $T(y, t)=W(y-c t)$, where $c$ is the speed of wave propagation [23]. This function satisfies the equation

$$
W^{\prime \prime}+c W^{\prime}+\Phi(W)=0
$$


(dependence of the function $\Phi$ on $y$ is omitted here) considered on the whole axis with the limits at infinity $W(-\infty)=T^{*}, W(\infty)=0$, where $T^{*}$ is the maximal solution of the equation $\Phi(T)=0$. Solution of equation (17) converges to the travelling wave solution if the initial condition is sufficiently large. The speed of the wave is positive if and only if the following condition is satisfied [24]:

$$
\int_{0}^{T^{*}} \Phi(T, y) d T>0
$$

Only positive velocity of thrombin wave propagation corresponds to the regime clot growth. Condition (18) also implies that thrombin production exceeds its loss due to its inhibition by antithrombin and blood flow.

Let us then consider the case of nonzero coefficients $k_{1}$ and $k_{2}$ and function $\Phi$ depending on $y$. If we assume that this dependence is weak, that is the derivative $\partial \Phi / \partial y$ is sufficiently small, we can use inequality (18) for each fixed value of $y$. Let us take the value of $y$ equal to zero that corresponds to the coordinate of the cell wall and then increase it considering the thrombin concentration on some positive distance from the wall. At some moment the inequality (18) will be no more satisfied and the critical value of $y$ will give an approximation of the maximal clot height.

Let us precise the conditions of the thrombin wave propagation (18) for the exact expression of function $\Phi$. At $y=0$, we have $B_{a}(0)=\lambda$ and $v(0)=0$, hence from (18) we get:

$$
A_{0}<\frac{1}{\alpha_{1}}\left(2 k_{1} \lambda \frac{T^{0}}{T^{*}}-k_{1} \lambda+k_{2} \frac{\left(T^{*}\right)^{2} T^{0}}{2}-2 k_{2} \frac{\left(T^{*}\right)^{3}}{5}\right)
$$

If this condition is satisfied, then the initial quantity of thrombin produced near the vessel wall is sufficiently large to initiate clot growth. Otherwise clot will not form. From the pathophysiological point of view this situation corresponds to bleeding, and it can occur for example in case of hemophilia or treatments with anticoagulant drugs. 


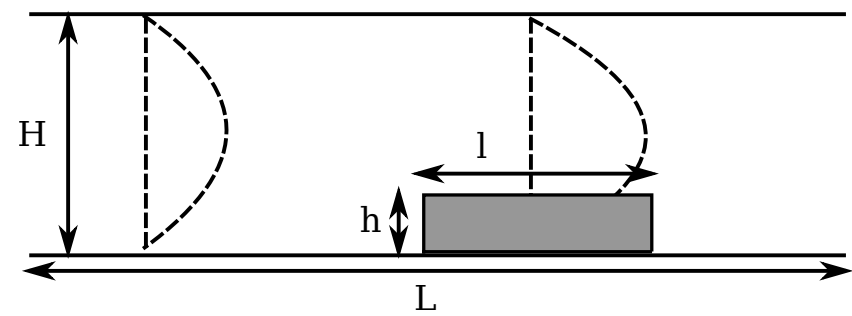

Figure 6. Schematic representation of a clot inside a blood vessel. The clot is shown in grey, possible blood flow profiles are described by dashed lines, geometrical parameters are shown.

\subsection{Flow velocity and clot growth}

Let us consider the simplified analytical model describing the interaction of the blood flow and growing clot. We consider the same computational domain as before and approximate the clot with a rectangle of length $l$ and height $h$ (Figure 6). To describe analytically the velocity profile, we consider blood flow as a simplified Poiseuille flow with the velocity $\vec{v}=(v(y), 0)$ determined by the equation:

$$
\frac{d^{2} v}{d y^{2}}=\frac{1}{\mu} \frac{d p}{d x}
$$

where is the kinematic viscosity and $\frac{d p}{d x}$ is the pressure gradient. Under the assumption that the clot permeability is low, the flow velocity at the clot-flow interface is described by the slip-flow boundary condition [25]. The solution of this equation is given by the truncated parabolic flow:

$$
v_{1}(y) \simeq\left\{\begin{array}{rl}
a y(H-y) & \text { if } y>h \\
0 & \text { if } y \leq h
\end{array},\right.
$$

where $H$ is the diameter of the vessel. We also consider Poiseuille flow in the part of the vessel without clot, $v_{2}(y)=b y(H-y)$. From the law of mass conservation, we get the equality:

$$
\int_{0}^{H} v_{1}(y) d y=\int_{0}^{H} v_{2}(y) d y .
$$

Taking the integrals, we obtain:

$$
a\left(\frac{(H-h)^{3}}{6}+\frac{h}{2}(H-h)^{2}\right)=b \frac{H^{3}}{6} .
$$


a. Velocity magnitude
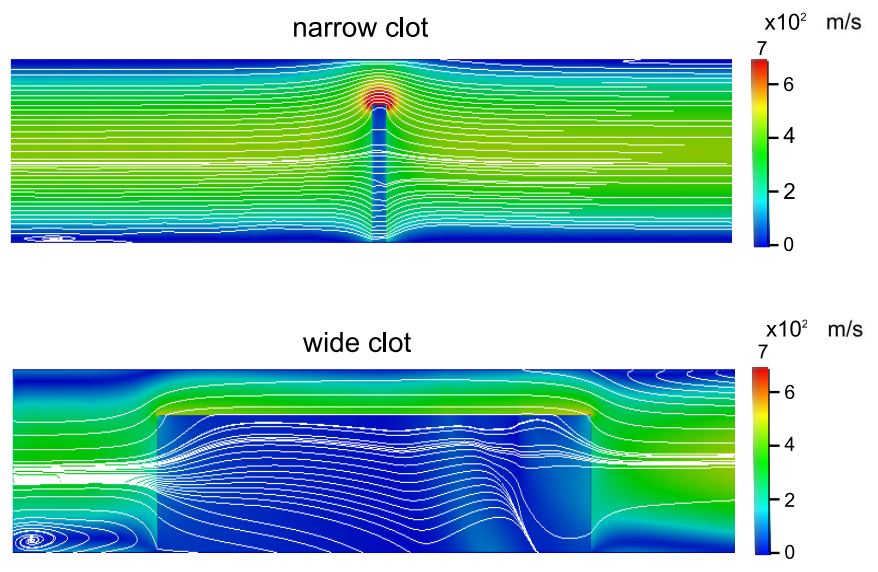

b. Velocity profile
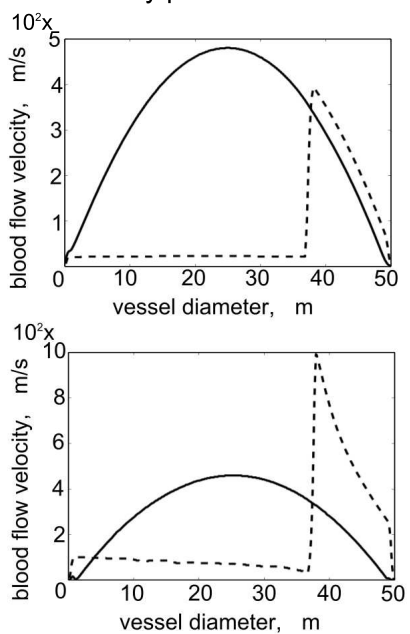

Figure 7. Numerical simulations of blood flow in the case of rectangular clots of different widths. a) 2D velocity profiles are shown with streamlines, b) Comparison between the initial parabolic profile (solid line) and the velocity profile at the vertical cross section in the middle of the clot (dashed line).

The total pressure drop equals the pressure difference at the narrow part of the channel (clot) and wide part of the channel (without clot):

$$
a l+b(L-l)=\beta,
$$

where $\beta=\frac{d p}{d x} L /(2 \mu)$. Together with the previous relation it allows us to determine $a$ :

$$
a=\frac{\beta}{l+(L-l)\left(1-\frac{h}{H}\right)^{2}\left(1+2 \frac{h}{H}\right)} .
$$

This coefficient depends on the clot width $l$ and its height $h$. In the limiting case $l=0, a$ is an increasing function of $h$ which tends to infinity as $h \rightarrow H$. For positive $l$ the function $a$ remains bounded with a parabolic type dependence (Figure 8, left) and the velocity decreases with increasing values of the clot width $l$. For small values of $l$ the maximum of $a$ is large enough to provide a high flow speed sufficient to stop clot grow that cannot be obtained for large $l$.

We can determine the final clot size integrating the condition (18): 


$$
v_{1}(y)<\frac{1}{\alpha_{2}}\left(2 k_{1} B_{a}(y) \frac{T^{0}}{T^{*}}-k_{1} B_{a}(y)+k_{2} \frac{\left(T^{*}\right)^{2} T^{0}}{2}-2 k_{2} \frac{\left(T^{*}\right)^{3}}{5}-\alpha_{1} A_{0}\right),
$$

where $v_{1}(y)$ is given by (21), (22). If this condition is satisfied, then the clot continues growing and it stops if the clot height $y$ is large enough to change the sign of the inequality. Hence we can determine conditions of partial or complete occlusion and the final clot size.

Let us recall that the maximal flow velocity $v_{m}$ at the entrance of the channel equals $b H^{2} / 4$. The dependence of the final clot size on this parameter is given in Figure 5, b. The two curves show the numerical solution of Equation (17) and the analytical approximation presented above. These curves are close to each other and their behavior is qualitatively similar to the dependence shown in Figure 5, a for the numerical solution of the complete problem. In both complete and simplified model we observe the complete vessel occlusion if the flow velocity is small enough and partial occlusion if the flow velocity is sufficiently large. These two regimes are separated with a sharp transition corresponding to the threshold values of the mean flow velocity.
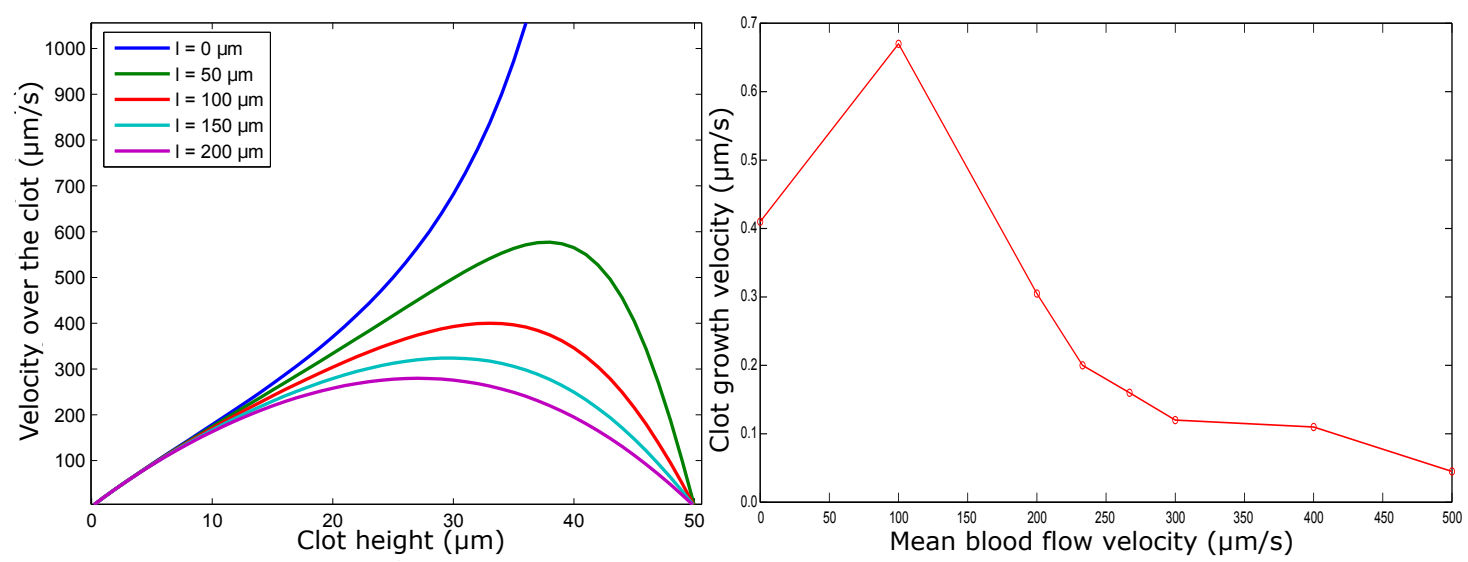

Figure 8. Left: maximal flow velocity in the analytical approximation (21) as a function of $h$. Right: clot growth velocity for different flow velocities in numerical simulations of the 2D problem. 


\section{DISCUSSION}

The main objective of the current study was to determine the conditions on the different regimes of vessel occlusion on the basis of mathematical models. In order to simulate chemical reactions of the coagulation cascade together with the blood flow dynamics we developed a mathematical model based on reaction-diffusion for the concentrations of blood factors and Navier-Stokes equations for the flow velocity. This model allowed us to identify the regimes of both partial and complete vessel occlusion and the conditions on the parameter values corresponding to these regimes. To understand the mechanisms underlying the observed phenomena we analyzed a simplified 1D model consisting of one reaction-diffusion equation for thrombin concentration.

We paid particular attention to the role of the blood flow in the inhibition of the clot growth. We used a pressure driven blood flow to model complete occlusion regimes and analyzed the change of the flow velocity profile for different sizes of the clot.

Clot growth speed in blood flow. The regimes of vessel occlusion identified in this work are also observed experimentally. Some of these experiments [26, 27] show that clot size depends on lumen diameter and blood flow. The effect of blood flow on propagation speed is studied in [28]. It is shown that the increase of blood flow velocity does not necessarily lead to a decrease of clot growth rate. In particular, three regimes can be identified: for low velocities, clot growth is stimulated by blood flow and the growth rate increases; for the intermediate flow velocities the rate of clot growth decreases until reaches a constant value; finally, for large flow velocities the rate of clot growth decays again.

In order to model this effect, we estimate the speed of clot growth in 2D numerical simulations using the relation:

$$
C=\frac{D_{10}}{t_{20}-t_{10}}
$$

where $t_{20}$ and $t_{10}$ are two time moments of time when the clot reaches the heights $20 \mu \mathrm{m}$ and 10 $\mu m$, respectively, and $D_{10}=10 \mu \mathrm{m}$. These two times are chosen in order to reduce the influence 
of the initiation phase and of APC inhibition. We represent the clot growth rate for different flow velocities in Figure 8 (right). Numerical simulations are in a good qualitative agreement with the experiments [28]. Quantitative difference with the experimental results can be related to the absence of platelets in the model studied in this work. Platelet aggregate slows down the flow at the injury site, and coagulation reactions can occur in this case for larger flow velocities than without platelets [16].

Limitations of the model. In this work we consider a rather complete but still a simplified model of clot growth in order to identify the main regimes of this process. We do not take into account platelets and some details of coagulation cascade. The preliminary simulations show that these factors can influence the quantitative conditions but the main regimes of clot growth remain the same. We will introduce them in the model in the forthcoming works together with the elasticity of vessel walls and pulsations of flow velocity.

\section{APPENDIX A: NUMERICAL METHODS AND COMPUTER IMPLEMENTATION}

The computational domain was discretized into a regular $2000 \times 400$ mesh. We used the finite difference method to implement the advection-diffusion-reaction equations and the projection method was used to solve the Navier-Stokes equation. These methods are briefly presented below.

\subsection{Advection-diffusion-reaction equations implementation}

Reaction-diffusion equations (e.g., Equation (2)) are discretized as follows:

$$
\begin{aligned}
\frac{T_{i, j}^{n+1}-T_{i, j}^{n}}{d t}+v_{x i, j}^{n}\left(T_{i, j}^{n}\right)_{x}^{+}+v_{y i, j}^{n}\left(T_{i, j}^{n}\right)_{y}^{+} & =D \frac{T_{i+1, j}^{n}+T_{i, j+1}^{n}-4 T^{n+1}+T_{i-1, j}^{n}+T_{i, j-1}^{n}}{h^{2}} \\
& +\left(\left(k_{3} B_{a i, j}^{n}\right)+\frac{k_{4}\left(T_{i, j}^{n}\right)^{3}}{1+k_{5} C_{a i, j}^{n}}\right) P_{i, j}^{n}-k_{a} A_{i, j}^{n} T_{i, j}^{n} .
\end{aligned}
$$

Here $d t$ the time step, $h$ is the space step as, $i$ and $j$ are point numbers, $n$ is the time step number. The terms $\left(T_{i, j}^{n}\right)_{x}^{+}$and $\left(T_{i, j}^{n}\right)_{y}^{+}$refers to an upwind scheme if the velocity is positive and to a downwind 
scheme otherwise. This scheme is conventionally introduced in order to treat numerical instabilities related to convection dominated problems. We have:

$$
\left(T_{i, j}^{n}\right)_{x}^{+}=\left\{\begin{array}{ll}
\frac{T_{i, j}^{n}-T_{i-1, j}^{n}}{h} & \text { if } v_{x i, j}^{n} \geq 0 \\
\frac{T_{i+1, j}^{n}-T_{i, j}^{n}}{h} & \text { if } v_{x i, j}^{n}<0
\end{array} .\right.
$$

The term $\left(T_{i, j}^{n}\right)_{y}^{+}$is treated in a similar way.

\subsection{Navier-Stokes equation implementation}

We solve the Navier-Stokes equations for the incompressible fluid

$$
\begin{gathered}
\frac{\partial u_{x}}{\partial t}+u_{x} \nabla \frac{\partial u_{x}}{\partial x}+u_{y} \nabla \frac{\partial u_{x}}{\partial y}=-\frac{\partial p}{\partial x}+\frac{1}{R e}\left(\frac{\partial^{2} u_{x}}{\partial x^{2}}+\frac{\partial^{2} u_{y}}{\partial x^{2}}\right)-\frac{1}{K_{f}} u_{x}, \\
\frac{\partial u_{y}}{\partial t}+u_{x} \nabla \frac{\partial u_{y}}{\partial x}+u_{y} \nabla \frac{\partial u_{y}}{\partial y}=-\frac{\partial p}{\partial y}+\frac{1}{R e}\left(\frac{\partial^{2} u_{x}}{\partial y^{2}}+\frac{\partial^{2} u_{y}}{\partial y^{2}}\right)-\frac{1}{K_{f}} u_{y} \\
\nabla \vec{u}=0
\end{gathered}
$$

using the Chorin projection method presented in [22]. The general approach is described in [29] (section 6.7). We proceed in three steps:

(i) Treat nonlinear terms: We look for an intermediate velocity $\left(u_{x}^{*}, u_{y}^{*}\right)$ verifying the equations:

$$
\begin{aligned}
& \frac{u_{x}^{*}-u_{x}^{n}}{d t}=-u_{x}^{n}\left(u_{x}^{n}\right)_{x}^{+}-u_{y}^{n}\left(u_{x}^{n}\right)_{y}^{+}-\frac{1}{K_{f}} u_{x}^{n}, \\
& \frac{u_{y}^{*}-u_{y}^{n}}{d t}=-u_{x}^{n}\left(u_{y}^{n}\right)_{x}^{+}-u_{y}^{n}\left(u_{y}^{n}\right)_{y}^{+}-\frac{1}{K_{f}} u_{y}^{n} .
\end{aligned}
$$

As above we use an upwind method to discretize the terms $\left(u_{x}^{n}\right)_{x}^{+},\left(u_{y}^{n}\right)_{x}^{+},\left(u_{x}^{n}\right)_{y}^{+},\left(u_{y}^{n}\right)_{y}^{+}$to avoid convection dominated problems.

(ii) Implicit viscosity: The viscosity terms are treated implicitly. We calculate a second intermediate velocity $\left(u_{x}^{* *}, u_{y}^{* *}\right)$ by solving the equations:

$$
\frac{u_{x}^{* *}-u_{x}^{*}}{d t}=\frac{1}{R e}\left(\left(u_{x}^{* *}\right)_{x x}+\left(u_{y}^{* *}\right)_{x x}\right), \quad \frac{u_{y}^{* *}-u_{y}^{*}}{d t}=\frac{1}{R e}\left(\left(u_{x}^{* *}\right)_{y y}+\left(u_{y}^{* *}\right)_{y y}\right) .
$$


(iii) Pressure correction: We correct the second intermediate velocity by the implicit pressure gradient $\left(\frac{\partial p^{n+1}}{\partial x}, \frac{\partial p^{n+1}}{\partial y}\right)$ :

$$
\frac{u_{x}^{n+1}-u_{x}^{* *}}{d t}=-\frac{\partial p^{n+1}}{\partial x}, \quad \frac{u_{y}^{n+1}-u_{y}^{* *}}{d t}=-\frac{\partial p^{n+1}}{\partial y} .
$$

The pressure gradient $\left(\frac{\partial p^{n+1}}{\partial x}, \frac{\partial p^{n+1}}{\partial y}\right)$ is only given implicitly since it is obtained by solving a linear system. In order to compute it, we solve the Poisson equation:

$$
-\Delta p^{n+1}=-\frac{1}{d t} \nabla \cdot u^{n}
$$

To solve the last equation, we use the successive over-relaxation method (SOR). It is an iterative method that converges faster than the Jacobi and Gauss-Seidel methods. In this method, we repeat the following iteration until the convergence of the solution:

$$
\begin{gathered}
p_{i, j}^{k+1}=(1-\omega) p_{i, j}^{k}+\frac{\omega}{4}\left(p_{i+1, j}^{k}+p_{i, j+1}^{k}+p_{i-1, j}^{k}+p_{i, j-1}^{k}\right)-\frac{h^{2}}{2 h d t}\left(u_{i+1, j}^{n}-\right. \\
\left.u_{i-1, j}^{n}+u_{i, j+1}^{n}-u_{i, j-1}^{n}\right),
\end{gathered}
$$

where the constant $\omega$ is the relaxation factor. Let us denote the number of points in the computational mesh by $N_{x} \times N_{y}$. To ensure the fast convergence of the SOR algorithm, we set

$$
\omega=2\left(1+\sqrt{1-\frac{\left(\cos \left(\pi / N_{x}\right)+\cos \left(\pi / N_{y}\right)^{2}\right.}{2}}\right)^{-1}
$$

\section{APPENDIX B: THE VALUES OF PARAMETERS}

The values of parameters used in numerical simulations for the 1D and 2D models are given in Table (I) and Table (II), respectively. Most of the reaction kinetics parameters are taken from [30, 31]. Concentrations are adimensionalized. Some parameters such as $\beta$ used in 1D model are estimated to fit the results of the $2 \mathrm{D}$ model. We consider the space unit $\mu \mathrm{m}=10^{-} 6 \mathrm{~m}$ and the time unit $t=10^{-} 2 \mathrm{~s}$. 


\begin{tabular}{|l|c|l|}
\hline Simulation & Numerical value & Description \\
\hline$d x$ & 0.2 & spatial step \\
$H$ & 0.02 & time step \\
$D$ & 50 & diameter of the vessel \\
\hline Reactions coefficients & Numerical value & Description \\
\hline$k_{1}$ & 1 & generation of thrombin in the initiation phase \\
$k_{2}$ & 22.5 & generation of thrombin in the propagation phase \\
$\alpha_{1}$ & 0.63 & thrombin-antithrombin binding reaction rate \\
$\alpha_{2}$ & 0.658 & thrombin removal rate by blood flow \\
\hline Physiological values & Numerical value & Description \\
\hline$A_{0}$ & 0.4 & antihtrombin concentration in blood plasma \\
$T_{0}$ & 0.8 & prothrombin concentration in blood plasma \\
\hline
\end{tabular}

Table I. The values of the parameters used in numerical simulations of the 1D model. 


\begin{tabular}{|c|c|c|}
\hline Simulation & Numerical value & Description \\
\hline$d x$ & 0.25 & spatial step \\
\hline$d t$ & 0.005 & time step \\
\hline$H$ & 50 & diameter of the vessel \\
\hline$L$ & 250 & length of the vessel \\
\hline$D$ & 0.5 & diffusion coefficient for all proteins \\
\hline Reactions coefficients & Numerical value & Description \\
\hline$k_{1}$ & 0.1 & conversion of fibrinogen into fibrin \\
\hline$k_{2}$ & 1 & generation of fibrin polymer from fibrin \\
\hline$k_{3}$ & 2500 & activation of prothrombin during the initiation phase \\
\hline$k_{4}$ & 22.5 & activation of prothrombin during the amplification phase \\
\hline$k_{5}$ & 3122.2 & inactivation of $\mathrm{V}$ by APC \\
\hline$k_{b}$ & 0.05 & IXa and Xa inactivation \\
\hline$k_{c}$ & 0.05 & APC inactivation \\
\hline$k_{7}$ & 40 & $T_{F}-V I I a$ binding with IXa and Xa \\
\hline$k_{8}$ & 9400 & $k_{8}=k_{9}^{-} k_{9}$ \\
\hline$k_{9}$ & 9400 & APC activation by thrombin-thrombomodulin \\
\hline$k_{a}$ & 0.68 & Inactivation of thrombin by antithrombin \\
\hline$k_{T}$ & 10000 & thrombin binding with thrombomodulin \\
\hline Physiological values & Numerical value & Description \\
\hline$V I I a$ & 0.5 & factor VIIa concentration on the damaged tissue \\
\hline$P_{0}$ & 0.8 & prothrombin concentration in blood plasma \\
\hline$A_{0}$ & variable & antihtrombin concentration in blood plasma \\
\hline$B_{0}$ & 1 & initial concenetrations of factors IX and X \\
\hline$C_{0}$ & 1 & initial concentration of APC \\
\hline$F_{g 0}$ & 1 & fibrinogen concentration in blood \\
\hline
\end{tabular}

Table II. The values of the parameters used in numerical simulations of the $2 \mathrm{D}$ model. 


\section{REFERENCES}

1. Esmon C T. The roles of protein C and thrombomodulin in the regulation of blood coagulation. J. Biol. Chem. 1989; 264(9):4743-4746.

2. Anand M, Rajagopal K, Rajagopal, K R. A model for the formation and lysis of blood clots. Pathophysiology of haemostasis and thrombosis 2005; 34(2-3):109-120.

3. Mann K G, Van't Veer C, Cawthern K, Butenas S. The role of the tissue factor pathway in initiation of coagulation. Blood coagulation \& fibrinolysis: an international journal in haemostasis and thrombosis 1998: 9:S3-7.

4. Fogelson A L, Tania N. (2005). Coagulation under flow: the influence of flow-mediated transport on the initiation and inhibition of coagulation. Pathophysiology of haemostasis and thrombosis 2005; 34(2-3):91-108.

5. Harrison S E, Bernsdorf J, Hose D R, Lawford P V. A lattice Boltzmann framework for simulation of thrombogenesis. Progress in Computational Fluid Dynamics, an International Journal 2008; 8(1-4):121-128.

6. Stortelder W, Hemker P W, Hemker H C. Mathematical modelling in blood coagulation; simulation and parameter estimation. Report-Modelling, analysis and simulation 1997; (20):1-11.

7. Galdi G P, Rannacher R, Robertson A M, Turek S. Hemodynamical flows. Modeling, analysis and simulation. Birkhäuser 2008, Basel.

8. Lobanov A I, Starozhilova T K. The effect of convective flows on blood coagulation processes. Pathophysiology of haemostasis and thrombosis 2005; 34(2-3):121-134.

9. Bessonov N, Sequeira A, Simakov S, Vassilevskii Yu, Volpert V. Methods of blood flow modelling. Mathematical Modelling of Natural Phenomena 2015; 10(1):1-25.

10. Tokarev A, Sirakov I, Panasenko G, Volpert V, Shnol E, Butylin A, Ataullakhanov F. Continuous mathematical model of platelet thrombus formation in blood flow. Russian Journal of Numerical Analysis and Mathematical Modelling 2012; 27(2),191-212.

11. Bodnr T, Sequeira A, Prosi M. On the shear-thinning and viscoelastic effects of blood flow under various flow rates. Applied Mathematics and Computation 2011; 217(11):5055-5067.

12. Leiderman K, Fogelson A L. Grow with the flow: a spatialtemporal model of platelet deposition and blood coagulation under flow. Mathematical Medicine and Biology 2010; dqq005.

13. Fogelson A L, Guy R D. Immersed-boundary-type models of intravascular platelet aggregation. Computer methods in applied mechanics and engineering 2008; 197(25):2087-2104.

14. Xu Z, Christley S, Lioi J, Kim O, Harvey C, Sun W, Alber M. Multiscale model of fibrin accumulation on the blood clot surface and platelet dynamics. Methods Cell Biol 2012; 110:367-388.

15. Bessonov N, Babushkina E, Golovashchenko S F, Tosenberger A, Ataullakhanov F, Panteleev M, Volpert V . Numerical modelling of cell distribution in blood flow. Mathematical Modelling of Natural Phenomena 2014; in print.

16. Tosenberger A, Ataullakhanov F, Bessonov N, Panteleev M, Tokarev A, Volpert V. Modelling of thrombus growth in flow with a DPD-PDE method. Journal of theoretical biology 2013; 337:30-41. 
17. Filipovic N, Kojic M, Tsuda A. Modelling thrombosis using dissipative particle dynamics method. Philosophical Transactions of the Royal Society of London A: Mathematical, Physical and Engineering Sciences 2008; 366(1879):3265-3279.

18. Tosenberger A, Ataullakhanov F, Bessonov N, Panteleev M, Tokarev A, Volpert V. Modelling of plateletfibrin clot formation in flow with a DPDPDE method. Journal of mathematical biology 2015; 1-33.

19. Bouchnita A, Tosenberger A, Volpert V. On the regimes of blood coagulation. Applied Mathematics Letters 2016; 51:74-79.

20. Wufsus A R, Macera N E, Neeves K B. The hydraulic permeability of blood clots as a function of fibrin and platelet density. Biophysical journal 2013; 104(8):1812-1823.

21. Neeves K B, Onasoga A A, Wufsus A R. The use of microfluidics in hemostasis: clinical diagnostics and biomimetic models of vascular injury. Current opinion in hematology 2013; 20(5): 417-423.

22. Chorin A J. Numerical solution of the Navier-Stokes equations. Mathematics of computation 1968; 22(104):745-762.

23. Volpert A I, Volpert V A, Volpert V A. Traveling wave solutions of parabolic systems (Vol. 140). American Mathematical Soc. 1994.

24. Volpert V. Elliptic Partial Differential Equations: Volume 2: Reaction-Diffusion Equations (Vol. 104). Springer 2014.

25. Beavers G S, Joseph D D. Boundary conditions at a naturally permeable wall. Journal of fluid mechanics 1967; 30(01):197-207.

26. Baumgartner H R (1973). The role of blood flow in platelet adhesion, fibrin deposition, and formation of mural thrombi. Microvascular research 1973; 5(2):167-179.

27. Falati S, Gross P, Merrill-Skoloff G, Furie B C, Furie B. Real-time in vivo imaging of platelets, tissue factor and fibrin during arterial thrombus formation in the mouse. Nature medicine 2002; 8(10):1175-1180.

28. Begent N, Born G V R. Growth rate in vivo of platelet thrombi, produced by iontophoresis of ADP, as a function of mean blood flow velocity. Nature 1970; 227: 926-930.

29. Strang G. Computational Science and Engineering, First Edition. Wellesley Cambridge 2007. Press.

30. Krasotkina Y V, Sinauridze E I, Ataullakhanov F I. Spatiotemporal dynamics of fibrin formation and spreading of active thrombin entering non-recalcified plasma by diffusion. Biochimica et Biophysica Acta(BBA)-General Subjects 2000; 1474(3):337-345.

31. Hockin M F, Jones K C, Everse S J, Mann K G. A model for the stoichiometric regulation of blood coagulation. Journal of Biological Chemistry 2002; 277(21):18322-18333. 